\title{
openheart Copper chelation in patients with hypertrophic cardiomyopathy
}

\author{
Anna Reid, ${ }^{1}$ Christopher Miller, ${ }^{1,2}$ John Peter Farrant (1) , ${ }^{1,2}$ Rahul Polturi, ${ }^{3}$ \\ David Clark, ${ }^{1}$ Simon Ray, ${ }^{1,2}$ Garth Cooper, ${ }^{2,4}$ Matthias Schmitt ${ }^{1}$
}

\begin{abstract}
- Additional supplemental material is published online only. To view, please visit the journal online (http://dx.doi.org/10.
\end{abstract} 1136/openhrt-2021-001803)

To cite: Reid A, Miller C, Farrant JP, et al. Copper chelation in patients with hypertrophic cardiomyopathy. Open Heart 2022;9:e001803. doi:10.1136/ openhrt-2021-001803

Received 5 August 2021 Accepted 15 November 2021

Check for updates

(C) Author(s) (or their employer(s)) 2022. Re-use permitted under CC BY-NC. No commercial re-use. See rights and permissions. Published by BMJ.

${ }^{1}$ Cardiovascular Division Northwest Heart Centre, Manchester University NHS Foundation Trust, Manchester UK

${ }^{2}$ Division of Cardiovascular Sciences, The University of Manchester, Manchester, UK ${ }^{3}$ ACALM Study Unit, Aston University, Birmingham, UK ${ }^{4}$ Division of Cardiovascular Sciences, Centre for Advanced Discovery and Experimental Therapeutics (CADET), The University of Manchester, Manchester, UK

Correspondence to Dr John Peter Farrant; john. farrant@mft.nhs.uk

\section{ABSTRACT}

Background Disturbances of copper $(\mathrm{Cu})$ homeostasis can lead to hypertrophic cardiac phenotypes (eg, Wilson's disease). We previously identified abnormal $\mathrm{Cu}$ homeostasis in patients with hypertrophic cardiomyopathy (HCM) and, therefore, hypothesised that $\mathrm{Cu}^{2+}$-selective chelation with trientine dihydrochloride may slow or reverse disease progression in HCM. The aim of this study was, therefore to explore the clinical efficacy, safety and tolerability of trientine in HCM.

Methods In this medicines and healthcare products regulatory agency (MHRA) registered open-label pilot study, we treated $20 \mathrm{HCM}$ patients with trientine for 6 months. Patients underwent a comprehensive assessment schedule including separate cardiac magnetic resonance imaging $(\mathrm{CMR})$ and $\mathrm{CMR}^{31} \mathrm{P}$-spectroscopy at baseline and end of therapy. Predefined end points included changes in left ventricular mass (LVM), markers of LV fibrosis, markers of LV performance and myocardial energetics. Ten matched patients with HCM were studied as controls.

Results Trientine treatment was safe and tolerated. Trientine caused a substantial increase in urinary copper excretion $(0.42 \pm 0.2$ vs $2.02 \pm 1.0, p=0.001)$ without affecting serum copper concentrations. Treatment was associated with significant improvements in total atrial strain and global longitudinal LV strain using both Echo and CMR. LVM decreased significantly in the treatment arm compared with the control group $(-4.2 \mathrm{~g} v 1.8 \mathrm{~g}$, $\mathrm{p}=0.03$ ). A strong trend towards an absolute decrease in LVM was observed in the treatment group $(p=0.06)$. These changes were associated with a significant change in total myocardial volume driven by a significant reduction in extracellular matrix (ECM) volume $(43.83 \pm 18.42$ $\mathrm{mL}$ vs $41.49 \pm 16.89 \mathrm{~mL}, \mathrm{p}=0.04$ ) as opposed to pure cellular mass reduction and occurred against a background of significant ECM volume increase in the control group $(44.59 \pm 16.50 \mathrm{~mL}$ vs $47.48 \pm 19.30 \mathrm{~mL}$, $\mathrm{p}=0.02)$. A non-significant $10 \%$ increase in myocardial phosphocreatine/adenosine triphosphate (PCr/ATP) ratio with trientine therapy $(1.27 \pm 0.44$ vs $1.4 \pm 0.39)$ was noted. Conclusions $\mathrm{Cu}^{2+}$-selective chelation with trientine in a controlled environment is safe and a potential future therapeutic target. A phase $2 \mathrm{~b}$ trial is now underway.

\section{INTRODUCTION}

Hypertrophic Cardiomyopathy (HCM), the most common monogenetic inherited cardiac disorder affecting 1 in every 500 of

\section{Key questions}

What is already known about this subject?

- Disturbances of copper homeostasis can lead to hypertrophic cardiac phenotypes, and it has previously identified that patients with hypertrophic cardiomyopathy (HCM) can have abnormal copper homeostasis.

What does this study add?

- The use of the copper chelator Trientine in patients with HCM is safe and well tolerated.

- Treatment was associated with improvements in many pathophysiological imaging biomarkers of HCM.

How might this impact clinical practice?

- This study has led to the 'randomised, double-blind placebo-controlled, phase 2 evaluation of the efficacy and mechanism of trientine in patients with hypertrophic cardiomyopathy (TEMPEST)' clinical trial for further evaluation of this drug in the HCM population.

the population. ${ }^{1}$ It is characterised by left ventricular (LV) hypertrophy $(\mathrm{LVH})$. Histologically, HCM displays myocyte enlargement and disarray, increased interstitial and replacement fibrosis and dysplastic intramyocardial arterioles.

Despite advances in diagnosis and risk stratification, there remains no licensed diseasemodifying treatment for HCM. Indeed, since HCM was first described, there have been less than 50 pharmacological studies, enrolling less than 2500 patients. In 2010, a National Institutes of Health working group identified a 'critical need for future research considerations directed toward basic and translational studies to identify putative targets for intervention. When viable targets are identified, clinical studies should be undertaken to determine if novel (or currently available) drug, or other therapies, can target these pathways of HCM disease expression and, improve on the natural history of patients with this disease. ${ }^{2}$

Copper $(\mathrm{Cu})$ is an essential cofactor for a variety of metabolic functions and while 
regulation of systemic Cu metabolism is critical to human health, ${ }^{3}$ free $\mathrm{Cu}$ ions are extremely redox-active and, thus, potentially toxic. ${ }^{4}$ Defective Cu metabolism is said to impair cardiovascular health in at least two known settings; chronic Cu deficiency and defective intracellular $\mathrm{Cu}$ transport to mitochondrial cytochrome-C oxidase. $\mathrm{Cu}$ excess is seen in Wilsons's disease and Chagas Cardiomyopathy, ${ }^{5}{ }^{6}$ with both conditions being characterised by LVH, and indeed both mimicking HCM. Cu deficiency, on the other hand, has been linked to the development of various phenotypes of cardiomyopathy. ${ }^{7}$ Simplistically, one can summarise that disturbances of $\mathrm{Cu}$ homeostasis can lead to both dilated and hypertrophic forms of cardiomyopathy and that $\mathrm{Cu}$ chelation, in the absence of $\mathrm{Cu}$ deficiency, is may be able to reverse LVH and organ fibrosis via a $\mathrm{Cu}$-dependent mechanism that reduces oxidative stress as demonstrated by us previously, both in animal and human studies. ${ }^{8}$

In a prospective observational cohort study of $20 \mathrm{HCM}$ patients and 18 matched healthy volunteers, we found that HCM was associated with significantly elevated serum copper and caeruloplasmin. ${ }^{9}$

Trientine dihydrochloride is a copper-chelating agent licensed for Wilson disease. In a randomised placebocontrolled trial in patients with type II diabetes and LVH, trientine was associated with a significant reduction in LV mass (LVM) without changes in blood pressure (BP) or glucose. ${ }^{10}$ Cumulative urine copper excretion was independently associated with the observed decrease in LVM.

In the present study, we aimed to explore the safety, tolerability and efficacy of trientine dihydrochloride in patients with HCM.

\section{METHODS}

\section{Study design}

This was an open-label single-site pilot study including 20 patients with HCM. Written, informed consent was obtained from all participants.

Ten additional age and gender-matched patients with HCM were recruited to a parallel 'natural-history-control' observational study to allow comparison of baseline and end-point data (ref: 14/NW/0060). Recruitment criteria were identical to those for the pilot study.

\section{Patient Selection}

Inclusion criteria were as follows: (1) age $\geq 18$ years, (2) a clinical diagnosis of HCM in line with the 2011 American College of Cardiology Foundation/American Heart Association consensus document, (3) LV ejection fraction $\geq 50 \%$. Exclusion criteria are shown listed in the supplementary data (online supplemental table S1).

\section{Intervention}

Patients received trientine dihydrochloride $300 \mathrm{mg}$ capsules at an initial dose of $300 \mathrm{mg}$ two times per day, which was increased after 1 week to $600 \mathrm{mg}$ two times per day if tolerated. Trientine was given for 6 months. Dose reductions were permitted in patients experiencing an adverse event. Patients were asked to return remaining capsules at each visit to assess compliance.

\section{Study outline visit schedule}

Online supplemental figure 1 in the supplementary data shows a study design flowchart. It was initially intended to schedule study visits at approximately 1 month, 2-month and 3-month intervals, however, for ease of dispensing of bottles of trientine (one bottle $=25$ days supply) and to limit time away from refrigeration, patients were followed up at 25-day multiples, without affecting study safety or integrity. No formal assessment of quality of life was performed.

Patients in the observational control study underwent cardiac magnetic resonance imaging (CMR) and phosphorous cardiac magnetic resonance spectroscopy $\left({ }^{31} \mathrm{P}-\right.$ CMRS) baseline and again at 6 months to match the trientine pilot study.

\section{Cardiac magnetic resonance}

Patients underwent CMR scanning at 1.5T (Avanto, Siemens AG, Erlangen, Germany). A dedicated and standardised HCM-CMR protocol was used in all. Steadystate free precession (SSFP) cine images were acquired in standard long-axis views. A stack of short-axis slices covered the left ventricle (LV), and left atrium (LA) for volumetric analysis. LV mass and volumetric analysis were performed using dedicated offline software (CMR Tools, Cardiovascular Imaging Solutions, London, UK).

Pre-contrast T1 mapping (Siemens MOLLI WIPP 448) was performed in the basal and mid short axis slices (chosen from the SSFP cines stack), four-chamber and three-chamber view. T2 mapping and T2* sequences were performed at the mid ventricular level. A circumferential region of interest was drawn to include the mid-LV wall, with care taken to avoid blood pool. Minimum, maximum and mean $\mathrm{T} 1$ values were noted. A blood sample was taken prior to CMR to measure serum haematocrit. Standard late gadolinium enhancement imaging (including phase-sensitive inversion-recovery) was performed at least $10 \mathrm{~min}$ after the final dose of contrast agent (Dotarem $0.15 \mathrm{mmol} / \mathrm{kg}$ ). Postcontrast T1 mapping was performed exactly $15 \mathrm{~min}$ after contrast administration. Extracellular volume (ECV) fraction, total myocardial volume, extracellular matrix (ECM) volume and cellular volume were calculated as previously described. ${ }^{11}$

Atrial and ventricular deformation/strain analysis were performed using commercially available feature tracking technology (TOMTEC Diogenes).

\section{P-cardiac MR spectroscopy}

${ }^{31} \mathrm{P}$-CMRS is an advanced MRI technique, which allows for the non-invasive, in vivo assessment of components of energy handling within myocardium. All patients underwent $3 \mathrm{~T}{ }^{31} \mathrm{P}$-CMRS at visits 2 and 6 (Achieva TX, Phillips, Best, Netherlands) using a dedicated, commercially available ${ }^{31} \mathrm{P}$ - transmit and receive coil $(14 \mathrm{~cm})$. Scans were performed with the patient lying prone. The protocol 
included acquiring survey images taken to assess the position of the mid interventricular septum relative to the centre of the coil, and the patient was repositioned as required to ensure minimal distance between the centre coil and isocentre of the magnet. Two ${ }^{31} \mathrm{P}$-spectra were acquired using a standard manufacturer supplied

${ }^{31}$ P-spectroscopy sequence. Spectra were summed and analysed on jMRUI software, with the PCr peak used as the reference peak. Quantification was first performed using the AMARES (Advanced Method for Accurate, Robust and Efficient Spectral fitting) fitting programme, in which the peaks and line widths of 2,3- diphosphoglycerate, PCr, and $\alpha, \beta$ and $\gamma$-ATP were automatically determined, and corrected for blood contamination as previously described. ${ }^{12}$ Area under the peak was used to determine the concentrations of metabolites, and myocardial phosphocreatine/adenosine triphosphate (PCr/ATP) ratio was subsequently calculated.

\section{Echocardiography}

Transthoracic echocardiography was performed on all patients using a GE vivid-9 machine. All measurements were made according to the American Society of Echocardiography guidelines and averaged over three cardiac cycles, during breath hold. Analysis was performed offline using customised software (EchoPac v113, GE Vingmed Ultrasound, Horten, Norway). Deformation analyses, that is, atrial and ventricular strain measurements, were based on speckle tracking technology.

All CMR, 31P CMRS and echocardiography analysis were performed by a Society of CMR and British Society of Echocardiography accredited operator blinded to patient data.

\section{Cardiopulmonary exercise testing}

Patients underwent symptom-limited (graded according to modified Borg scale) respiratory gas-exchange treadmill cardiopulmonary exercise testing. Tests were carried out using a modified Bruce protocol and the target respiratory exchange ratio (RER) was $>1.1$ for all studies. All tests were performed on the same system supervised by the same physician and analysed in a blinded fashion by a single experienced chief physiologist.

\section{Adverse events}

Adverse events assessed by the study investigators were recorded and followed up until resolution.

\section{Outcome measures}

Outcome measures were predefined as change in: (1) LVM, (2) markers of myocardial fibrosis (ie, native T1 and ECV), (3) LV myocardial deformation, (4) left atrial (LA) function, (5), myocardial energetics (PCr/ATP ratio), (6) exercise capacity.

\section{Statistical analysis}

Prespecified end points informing power calculation were change in LVM and PCr/ATP ratio after 6 months of treatment. Pilot work in patients with diabetes had revealed a mean reduction in $\mathrm{LVM}$ of $5 \mathrm{~g} / \mathrm{m}^{2}{ }^{12}$ with interobserver variability in our group of $10 \mathrm{~g} / \mathrm{m}^{2}$. Using this data, 20 patients treated provided an $80 \%$ power to detect changes in LVM/body surface area (BSA) of $4.6 \mathrm{~g} /$ $\mathrm{m}^{2}$ or more (using a paired t test with estimated SD of differences of $7 \mathrm{~g} / \mathrm{m}^{2}$ ) and changes of a PCr/ATP ratio of 0.36 or more (using a paired t test with estimated SD of differences of 0.55 ).

Power was also calculated for 15 patients, to allow for a $25 \%$ drop out rate (based on Conway et $a l^{12}$ ). The study then had $80 \%$ power to detect changes in LVM/BSA within the trientine group of $5.4 \mathrm{~g} / \mathrm{m}^{2}$ or more (using a paired $\mathrm{t}$ test with estimated SD of differences of $\left.7 \mathrm{~g} / \mathrm{m}^{2}\right)$.

Change in LVM and PCr/ATP ratio was used to inform power calculations. In the previously mentioned placebo controlled trial in diabetes, 6 months of treatment with trientine was associated with a within-group decrease in LVM indexed to body surface area (LVMi) of $5.0 \pm 7.2 \mathrm{~g}$ / $\mathrm{m}^{2}{ }^{12}$ Based on this data, 20 patients were required to provide $80 \%$ power for detecting a minimum difference in LVMi of $4.6 \mathrm{~g} / \mathrm{m}^{2}$ from baseline following 6 months of treatment, assuming an SD of $7 \mathrm{~g} / \mathrm{m}^{2}(5 \%$ significance level, two sided). Twenty patients would also provide $80 \%$ power for detecting a minimum difference in $\mathrm{PCr} / \mathrm{ATP}$ ratio of 0.36 , assuming an SD of 0.55 (5\% significance level, two sided).

Analysis was performed using SPSS V.23.0 (SPSS, Chicago, Illinois, USA). Data are presented as mean \pm SD Differences between groups were analysed using the paired or unpaired Student's t test, where appropriate. Statistical significance was defined as two-tailed $\mathrm{p}$ values $<0.05$.

\section{RESULTS}

\section{Study population}

Twenty-five patients consented to the pilot study of trientine but five were excluded prior to starting trientine: two patients were newly diagnosed with diabetes mellitus; one patient was found to have a large pleural effusion; one patient was found to be iron deficient and one patient had abnormal liver function tests.

Of the remaining 20 patients, 15 patients completed the study, with two requiring dose reduction to $600 \mathrm{mg}$ per day for the final 2 months of treatment, owing to gastrointestinal side effects. Four patients did not complete the study due to adverse effects and one patient withdrew due to a change in personal circumstance. Of the 10 patients who were recruited to the observational control group, one declined to return for follow-up and one declined the follow-up ${ }^{31} \mathrm{P}-\mathrm{CMRS}$. Both patients cited scan intolerability as the reason. Baseline patient characteristics are given in table 1.

\section{Safety of trientine}

Adverse events and their frequency are listed in the online supplemental tables S3, S4. There were four serious adverse events requiring hospitalisation: appendicitis 


\begin{tabular}{llll}
\hline Table 1 & Baseline patient characteristics & \\
\hline & $\begin{array}{l}\text { Intervention } \\
\text { group }\end{array}$ & $\begin{array}{l}\text { Control } \\
\text { group }\end{array}$ & P value* \\
\hline Age (years) & $55.5 \pm 8.4$ & $54.7 \pm 13.3$ & 0.86 \\
\hline Male & 15 & 7 & \\
\hline Body surface area $\left(\mathrm{m}^{2}\right)$ & $2.06 \pm 0.25$ & $1.94 \pm 0.24$ & 0.24 \\
\hline Systolic blood pressure $(\mathrm{mm} \mathrm{Hg})$ & $130 \pm 15$ & $137 \pm 13$ & 0.24 \\
\hline Diastolic blood pressure $(\mathrm{mm} \mathrm{Hg})$ & $81 \pm 12$ & $75 \pm 15$ & 0.28 \\
\hline Heart rate & $66.5 \pm 16.3$ & $65.1 \pm 11.5$ & 0.81 \\
\hline PCr/ATP ratio & $1.27 \pm 0.44$ & $1.51 \pm 0.82$ & 0.36 \\
\hline NYHA & 14 & 7 & \\
\hline NYHA II & 6 & 3 & \\
\hline EF (\%) & $69.0 \pm 6.8$ & $69.8 \pm 8.3$ & 0.79 \\
\hline Mass/BSA $\left(\mathrm{g} / \mathrm{m}^{2}\right)$ & $73.5 \pm 20.0$ & $79.8 \pm 18.6$ & 0.43 \\
\hline
\end{tabular}

${ }^{*}$ An expanded version of this table is available in the online supplemental table S2.

BSA, body surface area; EF, ejection fraction; NYHA, New York Heart association classification; PCr/ATP, myocardial phosphocreatine/ adenosine triphosphate.

after 1 week of treatment; palpitations with previous similar admissions; a recurrence of adhesive capsulitis requiring admission for analgesia; wrist sprain following a fall. None was judged to be related to trientine.

Trientine has been associated with iron deficiency, sideroblastic anaemia and leucopenia. There was no significant change in serum iron $(20.1 \pm 4.0 \mathrm{vs} 18.9 \pm 5.8 \mu \mathrm{mol} / \mathrm{L}$, $\mathrm{p}=0.4)$, haemoglobin $(145 \pm 13$ vs $143 \pm 13 \mathrm{~g} / \mathrm{L}, \mathrm{p}=0.4)$ or total white cell count $\left(6.9 \pm 1.3\right.$ vs $\left.7.0 \pm 1.6 \times 10^{9} / \mathrm{L}, \mathrm{p}=0.7\right)$ after 6 months of treatment. In addition, no clinically relevant change in renal or hepatic biochemistry or magnesium was observed.

\section{Efficacy of trientine}

\section{Biochemistry and haemodynamic data}

There was no change in serum copper or zinc after 6 months of trientine. There was a small but statistically significant increase in serum caeruloplasmin.

Compliance with 24-hour urinary copper collection was poor, particularly at the end of study visit (visit 6). Analysis was, therefore, conducted using data collected at visit 5 (150 days of treatment). Urinary copper increased significantly from baseline to visit $5(0.42 \pm 0.2$ vs $2.02 \pm 1.0 \mu \mathrm{mol} / \mathrm{L} / 24$ hours, $\mathrm{p}=0.001)$. Patients could continue in the study despite poor compliance with the 24-hour urine collections as change in urinary copper was not a primary outcome measure.

There were no clinically relevant changes in $\mathrm{BP}$, heart rate or body surface area with trientine (table 2).

\section{LVM and volumetrics}

Six months of trientine was associated with a strong trend towards a decrease in LVM. Indeed, in comparison to the observational control group, LVM decreased significantly $(-4.1 \pm 8.0 \mathrm{~g}$ vs $2.7 \pm 4.4 \mathrm{~g}, \mathrm{p}=0.03)$ (see online supplemental

\begin{tabular}{llll}
\hline Table 2 & Laboratory data & & \\
\hline & Baseline & Follow-up* & P value* \\
\hline Laboratory data & & & \\
Haemoglobin (g/L) & $145.5 \pm 13.3$ & $143.2 \pm 12.5$ & 0.40 \\
eGFR (mL/min/1.73 m²) & $82.7 \pm 8.6$ & $84.5 \pm 7.2$ & 0.30 \\
Alkaline phosphatase (IU/L) & $73.0 \pm 19.8$ & $78.6 \pm 19.1$ & 0.03 \\
ALT (IU/L) & $20.5 \pm 6.4$ & $23.6 \pm 5.0$ & 0.07 \\
Magnesium (mmol/L) & $0.87 \pm 0.08$ & $0.81 \pm 0.12$ & 0.14 \\
Serum copper $(\mu \mathrm{mol} / \mathrm{L})$ & $16.5 \pm 2.3$ & $16.6 \pm 2.7$ & 0.83 \\
Serum caeruloplasmin $(\mathrm{g} / \mathrm{L})$ & $0.23 \pm 0.03$ & $0.24 \pm 0.03$ & 0.04 \\
Haemodynamics & & & \\
Systolic BP (mm Hg) & $131 \pm 15$ & $125 \pm 15$ & 0.21 \\
Diastolic BP (mm Hg) & $83 \pm 14$ & $74 \pm 7$ & 0.06 \\
Heart rate (bpm) & $57.5 \pm 10.0$ & $58.8 \pm 9.2$ & 0.59 \\
\hline
\end{tabular}

*An expanded version of this table is available in the online supplemental table S5.

ALT, alanine transaminase; BP, blood pressure; eGFR, estimate glomerular filtration rate.

figure 2 in the supplementary data). No change was observed in $L V$ volumetrics (table 3 ).

An expanded version of this table is available in the supplementary data.

\section{CMR tissue characterisation}

Trientine treatment resulted in a reduction in native T1 $(1060 \pm 47 \mathrm{~ms}$ vs $1049 \pm 42 \mathrm{~ms})$ and ECV fraction $(30.0 \% \pm 4.5 \%$ vs $29.5 \pm 4.0 \%)$, approaching statistical significance ( $\mathrm{p}=0.06$ for both, online supplemental figure $3)$. By contrast, there was a significant increase in ECV fraction in the control arm $(29.0 \% \pm 5.1 \%$ vs $30.6 \pm 6.3 \%$, $\mathrm{p}=0.045$ ) over the $6 / 12$ study period. When determining compartmental myocardial changes based on these imaging parameters, trientine treatment was associated with a significant reduction in ECV, which occurred against a backdrop of a significant increase in ECM in the 'natural history' control group (see online supplemental figure 3 in the supplementary data).

There was a significant reduction in circumferential T2* (35.6 $\pm 4.1 \mathrm{~ms}$ vs $34.3 \pm 3.6 \mathrm{~ms}, \mathrm{p}<0.01$ ), a change not seen in the control group $(33.8 \pm 3.3 \mathrm{~ms}$ vs $33.0 \pm 3.9 \mathrm{~ms}$, $\mathrm{p}=0.58$ ). No difference in myocardial $\mathrm{T} 2$ was seen in either group.

\section{Left ventricular deformation}

There was significant improvement in global longitudinal strain, assessed both using feature tracking cardiac magnetic resonance imaging (FT-CMR) $(-18.3 \pm 3.4$ vs $-19.4 \pm 3.4, \mathrm{p}=0.03)$ and speckle-tracking echocardiography $(-16.6 \pm 4.3$ vs $-18.9 \pm 4.4, p=0.01)$. Septal S' velocity also significantly increased $(6.4 \pm 1.5 \mathrm{~cm} / \mathrm{s}$ vs $7.8 \pm 2.2 \mathrm{~cm} / \mathrm{s}$, $\mathrm{p}=0.04)$. By contrast, the control group displayed a significant reduction in FT-CMR derived global longitudinal strain $(-20.5 \pm 5.9$ vs $.-19.2 \pm 5.1, \mathrm{p}=0.01)$. No significant change was seen in circumferential strain in either group. 
Table 3 Cardiac MRI and echographic data at baseline and after 6 months of treatment with trientine

\begin{tabular}{|llll}
\hline & Baseline & Follow-up & P value \\
\hline Cardiac MRI: left ventricle & & & \\
\hline LVEDV (mL) & $171 \pm 36$ & $169 \pm 38$ & 0.57 \\
\hline LVESV (mL) & $54 \pm 20$ & $52 \pm 20$ & 0.41 \\
\hline SV (mL) & $117 \pm 22$ & $117 \pm 23$ & 0.98 \\
\hline EF (\%) & $69 \pm 7$ & $70 \pm 6$ & 0.55 \\
\hline LVM (g) & $152 \pm 54$ & $147 \pm 55$ & 0.06 \\
\hline Native septal T1 (ms) & $1060 \pm 47$ & $1049 \pm 42$ & 0.06 \\
\hline ECV fraction (\%) & $30.0 \pm 4.5$ & $29.5 \pm 4.0$ & 0.06 \\
\hline Total myocardial volume (mL) & $145 \pm 52$ & $140 \pm 53$ & 0.05 \\
\hline ECM volume (mL) & $44 \pm 18$ & $42 \pm 17$ & 0.04 \\
\hline Cellular volume (mL) & $101 \pm 36$ & $99 \pm 37$ & 0.11 \\
\hline Global longitudinal strain (\%) & $-18.3 \pm 3.4$ & $-19.4 \pm 3.4$ & 0.03 \\
\hline PCr/ATP ratio & $1.27 \pm 0.44$ & $1.4 \pm 0.39$ & 0.46 \\
\hline Cardiac MRI: left atrium & & & \\
\hline LAESV (mL) & $75.8 \pm 43.6$ & $65.8 \pm 40.9$ & 0.04 \\
\hline LAEDV (mL) & $124.4 \pm 51.3$ & $114.6 \pm 47.3$ & 0.18 \\
\hline Total EF (\%) & $41.3 \pm 8.3$ & $45.5 \pm 12.2$ & 0.07 \\
\hline LA expansion index & $0.73 \pm 0.22$ & $0.94 \pm 0.54$ & 0.09 \\
\hline Total strain (\%) & $20.0 \pm 3.9$ & $21.5 \pm 5.0$ & 0.04 \\
\hline Peak systolic strain rate (-1) & $0.75 \pm 13$ & $0.83 \pm 0.19$ & 0.11 \\
\hline Echocardiographic data & & & \\
\hline E/A ratio & $1.11 \pm 0.67$ & $1.10 \pm 0.50$ & 0.84 \\
\hline Mean S' velocity (cm/s) & $6.9 \pm 2.5$ & $8.1 \pm 1.9$ & 0.03 \\
\hline Mean E' velocity (cm/s) & $6.8 \pm 3.4$ & $7.0 \pm 2.5$ & 0.71 \\
\hline Mean A' velocity (cm/s) & $7.4 \pm 3.1$ & $8.4 \pm 2.7$ & 0.11 \\
\hline Mitral deceleration time (ms) & $295 \pm 79$ & $265 \pm 47$ & 0.11 \\
\hline & & & \\
\hline
\end{tabular}

ECV fraction was calculated as ECV $=(1$-haematocrit) $\times \lambda$, where $\lambda$ is the partition coefficient. Total myocardial volume was LV mass / 1.05. The total extracellular volume (ECM) was calculated with the formula $E C M=E C V \times L V M / 1.05$, while the cellular volume was calculated as cellular volume $(\mathrm{mL})=$ total myocardial volume - ECM volume. An expanded version of this table is available in the online supplemental tables S6, S7.

ECM, extracellular matrix; ECV, extracellular volume; EF, ejection fraction; LAEDV, left atrial end diastolic volume ; LAEF, left atrial ejection fraction; LVEDV, left vendricular end diastolic volume; LVESV, left ventricular end systolic volume ; LVM, left ventricular mass; PCr/ ATP, myocardial phosphocreatine/adenosine triphosphate; SV, stroke volume.

\section{Left atrial size and function}

There was a significant improvement in total left atrial strain using CMR-FT $\left(20.0 \pm 3.9^{-1}\right.$ vs $\left.21.5 \pm 5.0^{-1}, \mathrm{p}=0.04\right)$ and speckle-tracking echocardiography $\left(22.5 \pm 8.9^{-1} \pm 26.4\right.$ $\pm 8.5^{-1,} \mathrm{p}=0.03$ ). There was a corresponding trend towards improved left atrial ejection fraction $(41.3 \% \pm 8.3 \%$ vs . $45.4 \pm 12.2 \%, \mathrm{p}=0.06$ ) and reservoir expansion index $(0.73 \pm 0.22$ vs . $0.94 \pm 0.54, \mathrm{p}=0.09)$. Minimal $\mathrm{LA}$ volume significantly decreased $(75.8 \pm 43.6 \mathrm{~mL}$ vs $65.9 \pm 40.8 \mathrm{~mL}$, $\mathrm{p}=0.04$ ), with a trend towards reduced maximal volume $(124.3 \pm 51.3 \mathrm{~mL}$ vs $114.6 \pm 47.3 \mathrm{~mL}, \mathrm{p}=0.18)$. No significant change in left atrial strain was observed in the control group $\left(20.5 \pm 7.3^{-1}\right.$ vs $18.9 \pm 5.9$. $\left.\mathrm{p}=0.22\right)$.
Myocardial energetics

A typical ${ }^{31} \mathrm{P}-\mathrm{CMR}$ spectrum is shown in online supplemental figure 4 of the supplementary data. Mean Cramer Rao lower bounds (CRLB) for the whole group were $5.1 \%$ for PCr and $8.5 \%$ for ATP, representing satisfactory signal-to-noise ratio (SNR). ${ }^{13} 14$ One patient in both groups had CRLB greater than 20\% (previously reported to represent unacceptable SNR) ${ }^{1314}$ and was excluded. All remaining patients had CRLBs less than 20\%. No significant difference in $\mathrm{PCr} / \mathrm{ATP}$ ratio was found between those in New York Heart Association class I and II $(1.3 \pm 0.43$ vs $1.1 \pm 0.38, \mathrm{p}=0.29)$ at baseline. There was a small non-significant increase in mean $\mathrm{PCr} / \mathrm{ATP}$ ratio with trientine therapy $(1.27 \pm 0.44$ vs $1.4 \pm 0.39, \mathrm{p}=0.46)$.

\section{Exercise tolerance}

No significant change in exercise time, VO2 max or AT was observed (see online supplemental table S4 in the supplementary data). Target RER was not met by all participants during their examinations, however, there was no significant improvement seen in the effort-independent variables assessed.

\section{DISCUSSION}

In this open label pilot study, we found trientine to be safe and highly promising as a potential disease-modifying treatment for HCM.

LVH, myocardial fibrosis and impaired myocardial energetics are key pathophysiological features of HCM. There are currently no licensed therapies that target these disease processes in HCM, and, consequently, there are no pharmacological strategies that alter the natural history of HCM or reduce the associated risk of sudden death. Recently, the Explorer-HCM trial ${ }^{15}$ investigated the effects of a first-in class myosin inhibitor, mavacamten, in patients with symptomatic obstructive HCM. This trial demonstrated that treatment with mavacamtem resulted in improved exercise capacity, left ventricular outflow tract (LVOT) gradients and functional class as well as subsequently demonstrating LM mass regression in a selected substudy.

In this current study, trientine was observed to target key HCM disease manifestations. Short-term treatment with trientine was associated with a strong trend towards LVM regression. In comparison to an observational control group, it was associated with a significant reduction in LVM. The LVH regression was primarily driven by regression of myocardial fibrosis, assessed using CMR T1 and ECV, a central driver of morbidity and mortality in HCM. Trientine was also associated with a non-significant $10 \%$ improvement in myocardial energetics. Perhaps as a consequence of modifying the $\mathrm{LVH}$, myocardial fibrosis and energetics, trientine was associated with an improvement in atrial and longitudinal LV function. Furthermore, it can be postulated that the improvement in afterload-dependant measures such as LVH and LV strain 
may have contributed to the observed trend in improved diastolic BP.

Copper is an essential trace element found in all body tissues and has an important role in multiple biological functions, including signalling pathways, cellular respiration and as a cofactor for enzymatic activity. ${ }^{3}$ Within the human body, copper is found in its reduced state, $\mathrm{Cu}^{+}$and its oxidised state, $\mathrm{Cu}^{2+} \cdot \mathrm{Cu}^{2+}$ ions are the most highly redox-active metal ions in mammals, and as such are powerful catalysts of reactive oxygen species (ROS) and oxidative stress. ${ }^{4}$ Excess tissue $\mathrm{Cu}^{2+}$ ions lead to tissue damage by catalysing the formation of damaging hydroxyl $(\mathrm{OH} \cdot)$ radicals via the Fenton reaction. ${ }^{3}$ Furthermore, $\mathrm{Cu}^{2+}$ ions activate the transforming growth factor (TGF)betal signalling pathway, inhibit enzymatic antioxidants such as extracellular superoxide dismutase and augment the cardiotoxic effects of catecholamines. ${ }^{16}$ Experimentally, ROS are associated with myocardial hypertrophy, injury, apoptosis, necrosis and fibrosis. ${ }^{17} 18$ Furthermore, increased oxidative stress occurs in various models of $\mathrm{LV}$ pressure load and potentially perpetuates $\mathrm{LVH},{ }^{18-20}$ whereby cyclic strain causes an amplitude-related increase in ROS, associated with differential activation of kinases and induction of hypertrophic and apoptotic phenotypes. ${ }^{21}$

Recent research has highlighted the potential role of oxidative stress and low-grade inflammation in HCM pathophysiology. ${ }^{22}$ Dimitrow et al. found serum levels of 8-iso-prostaglandin F2 $\alpha$ (8-iso-PGF2 $\alpha$ ), a biomarker of generalised cellular oxidative stress, were elevated in 54 patients with HCM compared with matched controls. ${ }^{23}$ Patients with LVOT obstruction displayed higher 8-iso-PGF2a levels than those without LVOT obstruction. In a preclinical HCM study, N-acetylcysteine restored oxidised to total glutathione ratio, normalised levels of glutathiolated cardiac alpha-actin, restored myocardial levels of active protein kinase $\mathrm{G}$ and dephosphorylated nuclear factor of activated T cells 1 and phospho-p38, reversed cardiac and myocyte hypertrophy and interstitial fibrosis, reduced the propensity for ventricular arrhythmias and prevented cardiac dysfunction, ${ }^{24}$ although a recent clinical trial of $\mathrm{N}$-acetylcysteine in $\mathrm{HCM}$ was disappointing, although with a small sample size. ${ }^{25}$ In a transgenic model of human HCM, Senthil et al found atorvastatin reduced LVM, wall thickness, myocyte size, myocardial levels of molecular markers of hypertrophy, lipid peroxides and oxidised mitochondrial DNA. ${ }^{26}$

Accumulating data demonstrates a copper-overload state in diabetes and various forms of primary and secondary cardiomyopathies. ${ }^{67}$ Most recently, we demonstrated such a copper excess state to be present in HCM. ${ }^{9}$ Trientine has a range of actions thought to result from its removal of $\mathrm{Cu}^{2+}$ from tissue. We have demonstrated previously that trientine can reverse diabetes-induced damage to mitochondrial ultrastructure and normalise myocardial expression and enzymatic activity of proteins involved with energy metabolism, components of the mitochondrial respiratory chain and enzymes involved in fatty acid oxidation. ${ }^{27}$ This is relevant to HCM because energy depletion is hypothesised to be a primary disease mechanism, and inherited defects in mitochondrial energy production and fatty acid oxidation lead to phenotypes mimicking HCM.

Remarkably, we have demonstrated that trientine can prevent myocardial damage and result in tissue regeneration in the diabetes-damaged heart and kidney, including improvement in cardiomyocyte structure, myocardial collagen regression, LV function and alleviation of heart failure. $^{8} 1028$ We have also shown that trientine normalises extracellular superoxide dismutase, which inhibits ROS-mediated TGF-B activation and reverses myocardial fibrosis Importantly, when $\mathrm{Cu}^{2+}$ is removed from the body bound to trientine, it does not catalyse the Fenton reaction and so it does not cause tissue damage. The topic of $\mathrm{Cu}^{2+}$ selective chelation therapy for systemic diseases has been reviewed in greater detail elsewhere. ${ }^{29}$

Most relevant, in a randomised placebo-controlled trial in patients with type II diabetes and abnormal diastolic function, we previously demonstrated that trientine $1200 \mathrm{mg} /$ day was associated with a significant reduction in LVM. Cumulative urinary copper excretion independently associated with decrease in LVM. ${ }^{10}$ The current study replicates these results in patients with HCM. Taken together with the findings of our previous work and that of others, the beneficial effects of trientine on HCM pathophysiology observed in the current study may be mediated by an antioxidant effect of chelating myocardial $\mathrm{Cu} 2+$, leading to improved mitochondrial function and energy metabolism, and inhibition of TGF-B and profibrotic pathways. It is conceivable that by additionally targeting the accelerated fibrosis pathway in this disease, trientine may specifically address the progression of arrhythmic risk.

\section{Limitations}

This was an open-label pilot study and, thus, by its nature, was limited by its small sample size, limited duration and lack of a randomised placebo controlled parallel arm. Our patients were included on the basis of a clinical, not necessarily genetic, diagnosis. This represents a significant limitation to the study, however, it is important to note that currently, only approximately $60 \%$ o patient with clinical HCM have a confirmatory genetic mutation. ${ }^{30}$ It was necessary to conduct a proof of concept study in HCM before a definitive efficacy and mechanism evaluation could take place. In light of the findings of our prior study of trientine in type II diabetes, in which LVM regression continued to increase over the 12-month study period, it is not unreasonable to extrapolate that our 6-month study period was simply too short to elicit the full potential effect of trientine on the myocardium. These shortcomings are being addressed in TEMPEST (NCT04706429; www.tempest-trial.org.uk) an NIHR-EME (ref NIHR127575) sponsored appropriately powered randomised, double-blind, placebo-controlled phase $2 \mathrm{~b}$ multicentre trial currently under way. 
While the immediate test-retest variability of our ${ }^{31} \mathrm{P}$ CMRS method was good (intraclass correlation coefficient 0.932 in healthy volunteers), the 6 -month test-retest variability was simply too high (intraclass correlation coefficient 0.429 ) to permit reliable detection of trientineinduced changes with our sample size.

\section{CONCLUSION}

In this small open-label pilot study in HCM, trientine was found to be safe and well tolerated. Treatment was associated with improvements in many pathophysiological features of HCM most importantly LVM. Indeed, an appropriately powered phase $2 \mathrm{~b}$ double blinded, randomised multicentre study is now under way to answer whether trientine can slow or even halt HCM disease progression.

Contributors Study concept and design: MS, SR, CM, GC, AR, RP. Acquisition analysis or interpretation of data: all authors. Drafting, review and editing of manuscript: all authors. Study guarantor: MS.

Funding The study was funded by a MRC Confidence in Concept award. The investigational medicinal product was gifted by Univar. Univar had no role in the design and conduct of the study; collection, management, analysis and interpretation of the data; preparation, or approval of the manuscript; and decision to submit the manuscript for publication.

Competing interests $\mathrm{Dr}$ CM has received research support from Roche and Guerbet, served on an advisory board for Novartis and serves as an advisor for HAYA therapeutics.

Patient consent for publication Not applicable.

Ethics approval The study was approved by an ethics committee of the U.K. National Research Ethics Service (ref: 14/NW/1015) and the Medicines and Healthcare Products Regulatory Agency (21463/0220/001-0001) and performed in accordance with the Declaration of Helsinki.

Provenance and peer review Not commissioned; externally peer reviewed. Data availability statement Data are available upon reasonable request.

Open access This is an open access article distributed in accordance with the Creative Commons Attribution Non Commercial (CC BY-NC 4.0) license, which permits others to distribute, remix, adapt, build upon this work non-commercially, and license their derivative works on different terms, provided the original work is properly cited, appropriate credit is given, any changes made indicated, and the use is non-commercial. See: http://creativecommons.org/licenses/by-nc/4.0/.

ORCID iD

John Peter Farrant http://orcid.org/0000-0002-9241-2410

\section{REFERENCES}

1 Watkins $\mathrm{H}$, Ashrafian $\mathrm{H}$, McKenna WJ. The genetics of hypertrophic cardiomyopathy: Teare redux. Heart 2008;94:1264-8.

2 Force T, Bonow RO, Houser SR, et al. Research priorities in hypertrophic cardiomyopathy: report of a working group of the National heart, lung, and blood Institute. Circulation 2010;122:1130-3.

3 Uriu-Adams JY, Keen CL. Copper, oxidative stress, and human health. Mol Aspects Med 2005;26:268-98.

4 Zhang L, Ward M-L, Phillips ARJ, et al. Protection of the heart by treatment with a divalent-copper-selective chelator reveals a novel mechanism underlying cardiomyopathy in diabetic rats. Cardiovasc Diabetol 2013;12:123.

5 Burguera JL, Burguera M, Alarcón OM, et al. Concentration changes of zinc, copper and iron in serum of chronic chagasic myocardiopathic patients. J Trace Elem Electrolytes Health Dis 1988;2:215-9.

6 Hlubocká Z, Marecek Z, Linhart A, et al. Cardiac involvement in Wilson disease. J Inherit Metab Dis 2002;25:269-77.
7 Topuzoglu G, Erbay AR, Karul AB, et al. Concentrations of copper, zinc, and magnesium in sera from patients with idiopathic dilated cardiomyopathy. Biol Trace Elem Res 2003;95:11-18.

8 Cooper GJS, Phillips ARJ, Choong SY, et al. Regeneration of the heart in diabetes by selective copper chelation. Diabetes 2004;53:2501-8.

9 Potluri R, Roberts N, Miller C, et al. Impaired copper homeostasis in patients with hypertrophic cardiomyopathy. Eur Heart J 2012;33:741.

10 Cooper GJS, Young AA, Gamble GD, et al. A copper(II)-selective chelator ameliorates left-ventricular hypertrophy in type 2 diabetic patients: a randomised placebo-controlled study. Diabetologia 2009;52:715-22.

11 Flett AS, Hayward MP, Ashworth MT, et al. Equilibrium contrast cardiovascular magnetic resonance for the measurement of diffuse myocardial fibrosis: preliminary validation in humans. Circulation 2010;122:138-44.

12 Conway MA, Bottomley PA, Ouwerkerk R, et al. Mitral regurgitation: impaired systolic function, eccentric hypertrophy, and increased severity are linked to lower phosphocreatine/ATP ratios in humans. Circulation 1998;97:1716-23.

13 Shivu GN, Abozguia K, Phan TT, et al. (31)P magnetic resonance spectroscopy to measure in vivo cardiac energetics in normal myocardium and hypertrophic cardiomyopathy: Experiences at 3T. Eur J Radiol 2010;73:255-9.

14 Abozguia K, Elliott P, McKenna W, et al. Metabolic modulator perhexiline corrects energy deficiency and improves exercise capacity in symptomatic hypertrophic cardiomyopathy. Circulation 2010;122:1562-9.

15 Olivotto I, Oreziak A, Barriales-Villa R, et al. Mavacamten for treatment of symptomatic obstructive hypertrophic cardiomyopathy (EXPLORER-HCM): a randomised, double-blind, placebo-controlled, phase 3 trial. Lancet 2020;396:759-69.

16 Remião F, Carmo H, Carvalho F, et al. Copper enhances isoproterenol toxicity in isolated rat cardiomyocytes: effects on oxidative stress. Cardiovasc Toxicol 2001;1:195-204.

17 Minuz P, Fava C, Lechi A. Isoprostanes and vascular damage. Pharmacological Reports 2006;58:57-68.

18 Sawyer DB, Siwik DA, Xiao L, et al. Role of oxidative stress in myocardial hypertrophy and failure. $\mathrm{J} \mathrm{Mol} \mathrm{Cell} \mathrm{Cardiol}$ 2002;34:379-88.

19 Henderson BC, Tyagi N, Ovechkin A, et al. Oxidative remodeling in pressure overload induced chronic heart failure. Eur $J$ Heart Fail 2007;9:450-7.

20 Jacob MHVM, Pontes MRN, Araújo ASR, et al. Aortic-banding induces myocardial oxidative stress and changes in concentration and activity of antioxidants in male Wistar rats. Life Sci 2006;79:2187-93.

21 Pimentel DR, Amin JK, Xiao L, et al. Reactive oxygen species mediate amplitude-dependent hypertrophic and apoptotic responses to mechanical stretch in cardiac myocytes. Circ Res 2001;89:453-60.

22 Teekakirikul P, Eminaga S, Toka O, et al. Cardiac fibrosis in mice with hypertrophic cardiomyopathy is mediated by non-myocyte proliferation and requires TGF- $\beta$. J Clin Invest 2010;120:3520-9.

23 Dimitrow PP, Undas A, Wołkow P, et al. Enhanced oxidative stress in hypertrophic cardiomyopathy. Pharmacol Rep 2009;61:491-5.

24 Lombardi R, Rodriguez G, Chen SN, et al. Resolution of established cardiac hypertrophy and fibrosis and prevention of systolic dysfunction in a transgenic rabbit model of human cardiomyopathy through thiol-sensitive mechanisms. Circulation 2009;119:1398-407.

25 Marian AJ, Tan Y, Li L, et al. Hypertrophy regression with Nacetylcysteine in hypertrophic cardiomyopathy (HALT-HCM): a randomized, placebo-controlled, double-blind pilot study. Circ Res 2018;122:1109-18.

26 Senthil V, Chen SN, Tsybouleva N, et al. Prevention of cardiac hypertrophy by atorvastatin in a transgenic rabbit model of human hypertrophic cardiomyopathy. Circ Res 2005;97:285-92.

27 Jüllig M, Chen X, Hickey AJ, et al. Reversal of diabetes-evoked changes in mitochondrial protein expression of cardiac left ventricle by treatment with a copper(II)-selective chelator. Proteomics Clin Appl 2007;1:387-99.

28 Gong D, Lu J, Chen X, et al. A copper(II)-selective chelator ameliorates diabetes-evoked renal fibrosis and albuminuria, and suppresses pathogenic TGF-beta activation in the kidneys of rats used as a model of diabetes. Diabetologia 2008;51:1741-51.

29 Cooper GJS. Therapeutic potential of copper chelation with triethylenetetramine in managing diabetes mellitus and Alzheimer's disease. Drugs 2011;71:1281-320.

30 Marian AJ, Braunwald E. Hypertrophic cardiomyopathy: genetics, pathogenesis, clinical manifestations, diagnosis, and therapy. Circ Res 2017;121:749-70. 